\title{
Multimodal approaches to improve immunotherapy in breast cancer
}

\author{
Amrita Basu ${ }^{1}$ \& Krithika Kodumudi*,1,2 \\ ${ }^{1}$ Immunology Program \& Department of Clinical Science, H. Lee Moffitt Cancer Center, Tampa, FL 33612, USA \\ ${ }^{2}$ Department of Oncological Sciences, USF Morsani College of Medicine, University of South Florida, Tampa, FL 33612, USA \\ *Author for correspondence: krithika.kodumudi@moffitt.org
}

\author{
"Focusing future studies on other oncodrivers expressed in other BC subtypes will help to \\ develop oncodriver-targeted immunotherapy to stimulate a CD4 ${ }^{+}$Th1 immune response and to \\ design novel combination therapies"
}

First draft submitted: 7 November 2019; Accepted for publication: 12 February 2020; Published online: 11 March 2020

Keywords: cancer immunology $\bullet$ cancer vaccines $\bullet$ cell signaling $\bullet$ cellular immunology • checkpoint inhibitors $\bullet$ clinical immunology $\bullet$ cytokines $\bullet$ immunotherapy $\bullet$ oncodriver $\bullet$ Th1 $\bullet$ therapeutic vaccines

Breast cancer (BC) is the second most commonly detected cancer in women, with an estimated 268,600 new cases diagnosed in 2019 [1]. Resistance to targeted therapies and subsequent metastasis in BC urges researchers to develop novel strategies to overcome resistance. Cancer immunotherapy has been a promising approach for the treatment of $\mathrm{BC}$ [2]. However, various immunosuppressive mechanisms and immune checkpoint pathways in the tumor microenvironment (TME) have led to limited success in immunotherapy trials. In this editorial, we focus on oncodrivers, metabolism and tumor vascular architecture and their cross-talk with immune response in the TME, as well as how targeting such pathways may improve the therapeutic efficacy of immunotherapy in metastatic BC $(\mathrm{MBC})$.

\section{Oncodrivers in breast cancer}

Oncodrivers, in other words, receptor proteins that are critical for the proliferation and survival of cancer cells, have been studied due to their potential for targeted therapy development in BC. Overexpression of oncodrivers in cancer presents them as the quintessential tumor-associated antigens for driving antitumor immune response. In BC, overexpression of HER2/ERBB2, a member of the ERBB family of growth receptors, plays a critical role in tumorogenesis [3]. Constitutive HER2 activation is associated with proliferation, therapy resistance and metastasis in HER2 + BC [4-8]. Unlike luminal and HER2+ BCs, triple negative BC (TNBC) is marked by a lack of hormone receptors [9] and suffers from a lack of targeted therapy. Recent research has identified a range of oncodriver receptors overexpressed in TNBC tumors, namely HER3, EGFR and c-MET/HGFR that encourage further research into targeted therapy development. Oncodriver-targeted therapies in BC have impacted patient outcome significantly, however, therapy resistance and metastasis both remain challenges to successful patient outcome. Details of targeted therapy development, efficacy and resistance have been reviewed elsewhere $[7,10,11]$.

\section{Immunotherapy in breast cancer}

Immunotherapy in $\mathrm{BC}$ has garnered attention in recent years due to its promising therapeutic benefits observed in various other cancers $[12,13]$. In the TME, $\mathrm{CD}^{+}$and $\mathrm{CD}^{+} \mathrm{T}$ cell-induced antitumor immune response is balanced between CTL (cytotoxic T lymphocytes)-supportive and CTL-limiting CD4 ${ }^{+}$Th1 cytokines (IL-10, IL-4 and IL-5), with both influencing the success of immunotherapy [14,15]. CD4 ${ }^{+}$immune response induces IFN- $\gamma$ production and is critical for CD8 ${ }^{+}$CTL activity, enhanced activity of APCs and NK-cell function [6,16]. Suppressor cells such as myeloid-derived suppressor cells (MDSCs) and Tregs contribute to immuno-suppression by pivoting adaptive immune response to the Th2 phenotype [17]. Clinical success of dendritic cell (DC)-based targeted immunotherapy [18] suggests there is therapeutic potential for DCs across all BC subtypes. While poor 
immunogenicity and T-cell infiltration with immunosuppressive pathway activation have been identified as potential challenges to immunotherapy success in $\mathrm{BC}[19,20]$, a myriad of other factors in the TME synchronize the therapeutic response. Here we discuss how tumor oncodriver expression, metabolic reprogramming and altered tumor vasculature may subdue immune response in the TME. We will also consider how multimodal treatment strategy can overcome the current challenges of MBC.

While current immunotherapy primarily focuses on $\mathrm{CD} 8^{+} \mathrm{CTL}$ activity, our lab identified a progressive loss of HER2-specific $\mathrm{CD}^{+}$Th1 immune response in HER2+ BC patients' peripheral blood [15]. This suggests a crosstalk between oncodriver and immune response pathways in $\mathrm{BC}$ that could be critical for the growth, survival and metastasis of cancer cells. We demonstrated that a combination of Th1 cytokines (IFN- $\gamma$ and TNF- $\alpha$ ) increased cellular senescence in HER2+ BC cells and reversed resistance to anti-HER2 treatment [21]. In HER2+ BC patients, restoration of the lost anti-HER2 CD4 Th1 immune response by a mature, type-I polarized DC (DC1) vaccine pulsed with HER2 peptides resulted in an improved pathologic complete response [22]. Multiple ongoing clinical trials are investigating the therapeutic efficacy of the HER2-DC1 vaccine in the receptor positive primary and MBC landscape (NCT03384914 and NCT03112590). Additionally, a combination of HER2-DC1 vaccine with HER2 targeted therapy and checkpoint blockade significantly delayed tumor burden and improved survival in a preclinical HER2+ BC model [23]. Thus, a combinatorial approach to diminish HER2 oncodriver activity and trigger Th1 immune response will be required to overcome resistance to anti-HER2 therapeutics observed frequently in HER2+ BC and maximize patient benefit.

Similarly, our lab has reported a loss of HER3-specific Th1 immune response in peripheral blood from TNBC patients that correlated with poor outcome [24]. Considering the lack of targetable surface receptors in TNBC, HER3 as an oncodriver appears as a promising target for developing cellular immunotherapy. Shared intracellular signaling pathways between oncodrivers and the immune system suggests that a multi-dimensional blockade of such crosstalk will improve therapeutic success in BC. Immunotherapy and targeted therapy have shown promising therapeutic response individually in various BC subtypes. However, singular therapeutic approach may not be sufficient to tackle the complex regulation of immune cells and growth factor receptors in the TME. Targeting an oncodriver to generate antitumor immune response along with blockade of that specific oncodriver may yield superior therapeutic efficacy in BC, where oncodriver overexpression is a frequent phenomenon in the TME. We believe future research will shed light on the mutual interaction between oncodrivers and the immune system at the cellular level and will identify the optimal combinatorial therapy strategy for aggressive BCs like TNBC.

\section{Tumor vasculature \& immune response}

To meet the persistent demand for nutrients and oxygen in a proliferating tumor, continued production of proangiogenic factors (VEGF, TGF $\beta$, PDGF and FGF) tip the balance between pro- and anti-angiogenic factors, resulting in aberrant and leaky tumor vasculature formation characterized by loosely attached endothelial cells and low expression of VCAM1 and ICAM1 to inhibit immune cell infiltration [25]. These structural abnormalities may limit the success of immunotherapy in an otherwise immunologically 'cold' BC, characterized by low tumor infiltrating lymphocytes and activity [20]. An uneven distribution of blood vessels and impaired perfusion of the tumor cells lead to a highly hypoxic TME, which promotes accumulation of MDSCs and $\mathrm{T}_{\text {reg }}$, and the polarization of tumor-associated macrophages (TAMs) into the immunosuppressive $\mathrm{M} 2-\mathrm{M} \varphi$ phenotype, leading to suppressed effector function [26].

Bevacizumab, the monoclonal antibody against proangiogenic factor VEGF-A, has been approved by the US FDA for different cancer types [27] and the combination of bevacizumab and immunotherapy is currently being tested in various clinical trials (NCT03475004, NCT03367871 and NCT03526432). Among other angiogenic signaling axis, the ANG1-ANG2-TIE1-TIE2 axis has garnered interest since simultaneous ANG2 and VEGF blockade has been associated with more efficient antigen priming by APCs, TAM polarization to M1-M $\varphi$ phenotype and the infiltration of $\mathrm{CD}^{+} \mathrm{T}$ cells [28]. Notably, Semaphorin-4D (SEMA4D) has been identified as a promising modulator of tumor vasculature and a facilitator of an immunosupportive TME in solid tumors. SEMA4D has been shown to induce cytoskeletal changes in immune, vascular and tumor cells. Produced by the immunosuppressive MDSCs and $\mathrm{M} 2-\mathrm{M} \varphi$ TAMs within the TME, SEMA4D has been reported to inhibit immune cell movement, cause vascular destabilization and transactivate oncodrivers HER2 and c-MET [29,30]. Antibody-mediated SEMA4D neutralization results in increased macrophage infiltration and intratumoral $\mathrm{CD}^{+} \mathrm{T}$ cell, dispersion of MDSCs and $\mathrm{M} 2-\mathrm{M} \varphi$ TAMs, a pro-inflammatory cytokine milieu including enhanced IFN- $\gamma$ and TNF- $\alpha$ and reduced MCP-1 (chemoattractant for MDSCs) secretion [31,32]. A humanized anti-SEMA4D antibody, pepinemab, is 
currently being tested in multiple clinical trials for various cancers, in combination with PD1/PDL1 checkpoint inhibitors (NCT03320330, NCT03690986 and NCT03769155). Common regulators of immune response and angiogenesis suggest combinatorial therapies targeting these two pathways may impact TME in a synergistic manner to inhibit tumor growth. Considering this dynamic interaction between immune infiltration and response with the constantly evolving vasculature in a growing and treated tumor, combinatorial immunotherapy with antiangiogenic therapy will improve the chance of therapeutic benefit in resistant MBC and other cancers where monotherapy is not ideal.

\section{Metabolic reprogramming in breast cancer \& communication with immune response}

Metabolic reprogramming is the stress response of tumor cells by altering the metabolic profile through genetic mutations and epigenetic changes. Increased aerobic glycolysis, fatty acid synthesis and glutaminolysis to accommodate the steadily rising demand for essential nutrients are characteristics of most malignant cells including BC. Since Warburg's first postulate in 1956 regarding increased glycolysis in cancer cells and conversion of pyruvate to lactate rather than Acetyl-coA-A for ATP production (later known as Warburg effect), metabolic alteration has been linked to therapy resistance and immune escape in $\mathrm{BC}$ [33]. A higher glutamate-to-glutamine ratio and frequent expression of key regulatory enzymes [34] in HER2+ and TNBC indicate upregulated glutamine metabolism, another hallmark of metabolic reprogramming. On the other hand, a bidirectional regulatory network between HER2 and fatty acid synthase (FASN) has been suggested to contribute to upregulated fatty acid synthesis observed in BC [35,36] (NCT03179904). Hypoxia and tumor cell necrosis enhance the extracellular concentration of immune inhibitory metabolites adenosine and lactate. Lactate accumulation leads to metabolic lactic acidosis, resulting in impaired CTL by interfering with IFN- $\gamma$ production [37]. HIF-1 $\alpha$ induced by hypoxia activates the PI3K pathway, which is critical for glucose metabolic reprogramming [36].

Metabolic rewiring is a characteristic of immune activation and plays a critical role in determining immunotherapy success in the TME [38]. Lymphoid cells switch to aerobic glycolysis from oxidative phosphorylation (OXPHOS) for activation, while high glycolytic flux is critical for $\mathrm{DC}$ and $\mathrm{M} 1-\mathrm{M} \varphi$ activation [39]. Changes in the $\mathrm{pH}$ gradient by lactate release and extracellular matrix (ECM) acidification accumulates lactate in the T cell, leading to T-cell anergy and diminished proliferation [33]. Overexpression of indoleamine 2,3-dioxygenase (IDO) on tumor cells and immune cells results in tryptophan deprivation, T-cell anergy and reduced proliferation, along with lower $\mathrm{CD}^{+}$T-cell infiltration to create de novo immunosuppression [40]. IDO-expressing DCs induce the tolerogenic phenotype by stimulating IDO expression and the production of kynurenin, a soluble tryptophan catabolite, which inhibits IL-12, promotes IL-10 and TGF- $\beta$ secretion and alters the antigen presentation capacity of DCs to the tolerogenic phenotype [41]. Kynurenine also induces Treg production and stimulates apoptosis in CD8 ${ }^{+}$ TIL and CD4 ${ }^{+}$Th1 cells, but not in Th2 cells [33,40,42]. These studies re-affirm the interactive network between proliferative signaling, vasculature and immune response in the TME. Immune response generated as a direct or bystander effect of therapeutics that regulates tumor metabolic switch can be crucial in determining success of tumor immunotherapy. Simultaneously, metabolic re-wiring by immunotherapy can contribute to a synergistic antitumor response. Therefore, the combination of immunotherapy and metabolic inhibitors may improve therapeutic efficacy in BC patients, compared with monotherapy. We believe future research will help in understanding the molecular details of the signaling cross-talk between metabolic drivers and immune cells in the TME, identifying an optimal combinatorial treatment strategy for BC.

Along with targeting TME modulators as discussed above, the combination of multimodal immunotherapy may enhance antitumor immune response and improve outcome. Several clinical trials are currently investigating the therapeutic efficacy of combination of immune checkpoint inhibitors with HER2-targeted therapy in various HER2+ BC patient cohorts (NCT03387553, NCT03747120 and NCT03199885). Focusing future studies on other oncodrivers expressed in other BC subtypes will help to develop oncodriver-targeted immunotherapy to stimulate a CD4 ${ }^{+}$Th1 immune response and to design novel combination therapies. Immune modulating effects of standard-of-care therapeutics, such as immunogenic cell death leading to DC activation and increased CD8 ${ }^{+}$ T-cell proliferation by anthracycline, increased TIL recruitment and decreased MDSCs by taxane and increased MHC-I expression on tumors by platinum salts (BMC2019) [43], suggest their combination with immunotherapy may enhance therapeutic efficacy. 


\section{Conclusion}

Although many targeted therapies have shown to induce antitumor immune response in $\mathrm{BC}$, the response is often impeded by a myriad of factors present in the TME and their regulatory interplay. Given the complexity of the immune response to cancer, targeting one pathway could lead to an escape mechanism in tumor cells and contribute to monotherapy resistance. Therefore, we emphasize on targeting oncodriver signaling, vasculature redesigning and metabolic reprogramming pathways to prevent feedback loops. With an increasing number of immunotherapeutic agents available clinically, understanding these networks will open new avenues for better clinical outcome and ultimately will result in improved combinatorial approaches in BC.

\section{Financial \& competing interests disclosure}

The authors have no relevant affiliations or financial involvement with any organization or entity with a financial interest in or financial conflict with the subject matter or materials discussed in the manuscript. This includes employment, consultancies, honoraria, stock ownership or options, expert testimony, grants or patents received or pending, or royalties.

No writing assistance was utilized in the production of this manuscript.

\section{References}

1. Society AC. Breast cancer facts \& figures 2019-2020. Breast Cancer Statistics 2019 (2019). www.cancer.org/research/cancer-facts-statistics/breast-cancer-facts-figures.html

2. Adams S, Gatti-Mays ME, Kalinsky K et al. Current landscape of immunotherapy in breast cancer: a review. JAMA Oncol. doi:10.1001/jamaoncol.2018.7147 (2019) (Epub ahead of print).

3. Harbeck N, Penault-Llorca F, Cortes J et al. Breast cancer Nat. Rev. Dis. Primers 5(1), 66 (2019).

4. Escriva-De-Romani S, Arumi M, Bellet M, Saura C. HER2-positive breast cancer: current and new therapeutic strategies. Breast 39, 80-88 (2018).

5. Holgado E, Perez-Garcia J, Gion M, Cortes J. Is there a role for immunotherapy in HER2-positive breast cancer? NPJ Breast Cancer 4, 21 (2018).

6. Nocera NF, Lee MC, De La Cruz LM, Rosemblit C, Czerniecki BJ. Restoring lost anti-HER-2 Th1 immunity in breast cancer: a crucial role for Th1 cytokines in therapy and prevention. Front. Pharmacol. 7, 356 (2016).

7. Oh DY, Bang YJ. HER2-targeted therapies - a role beyond breast cancer. Nat. Rev. Clin. Oncol. 17(1), 33-48 (2019).

8. Triulzi T, Forte L, Regondi V et al. HER2 signaling regulates the tumor immune microenvironment and trastuzumab efficacy. Oncoimmunology 8(1), e1512942 (2019).

9. Sotiriou C, Neo SY, Mcshane LM et al. Breast cancer classification and prognosis based on gene expression profiles from a population-based study. Proc. Natl Acad. Sci. USA 100(18), 10393-10398 (2003).

10. Berzofsky JA, Terabe M, Trepel JB et al. Cancer vaccine strategies: translation from mice to human clinical trials. Cancer Immunol. Immunother. 67(12), 1863-1869 (2018).

11. Martinez MT, Perez-Fidalgo JA, Martin-Martorell P et al. Treatment of HER2 positive advanced breast cancer with T-DM1: a review of the literature. Crit. Rev. Oncol. Hematol. 97, 96-106 (2016).

12. Basu A, Ramamoorthi G, Jia Y et al. Immunotherapy in breast cancer: current status and future directions. Adv. Cancer Res. 143, 295-349 (2019).

13. Sanmamed MF, Chen L. A paradigm shift in cancer immunotherapy: from enhancement to normalization. Cell 175(2), 313-326 (2018).

14. Borst J, Ahrends T, Babala N, Melief CJM, Kastenmuller W. CD4(+) T cell help in cancer immunology and immunotherapy. Nat. Rev. Immunol. 18(10), 635-647 (2018).

15. Datta J, Fracol M, Mcmillan MT et al. Association of depressed anti-HER2 T-Helper type 1 response with recurrence in patients with completely treated HER2-positive breast cancer: role for immune monitoring. JAMA Oncol. 2(2), 242-246 (2016).

16. Schietinger A, Philip M, Liu RB, Schreiber K, Schreiber H. Bystander killing of cancer requires the cooperation of CD4(+) and CD8(+) T cells during the effector phase. J. Exp. Med. 207(11), 2469-2477 (2010).

17. Gonda K, Shibata M, Ohtake T et al. Myeloid-derived suppressor cells are increased and correlated with type 2 immune responses, malnutrition, inflammation, and poor prognosis in patients with breast cancer. Oncol. Lett. 14(2), 1766-1774 (2017).

18. Burke EE, Kodumudi K, Ramamoorthi G, Czerniecki BJ. Vaccine therapies for breast cancer. Surg. Oncol. Clin. N. Am. 28(3), 353-367 (2019).

19. Sambi M, Bagheri L, Szewczuk MR. Current challenges in cancer immunotherapy: multimodal approaches to improve efficacy and patient response rates. J. Oncol. 2019, 4508794 (2019).

20. Sharma P, Allison JP. The future of immune checkpoint therapy. Science 348(6230), 56-61 (2015). 
21. Rosemblit C, Datta J, Lowenfeld L et al. Oncodriver inhibition and CD4(+) Th1 cytokines cooperate through stat1 activation to induce tumor senescence and apoptosis in HER2+ and triple negative breast cancer: implications for combining immune and targeted therapies. Oncotarget 9(33), 23058-23077 (2018).

22. Lowenfeld L, Mick R, Datta J et al. Dendritic cell vaccination enhances immune responses and induces regression of HER2(pos) DCIS independent of route: results of randomized selection design trial. Clin. Cancer Res. 23(12), 2961-2971 (2017).

23. Kodumudi KN, Ramamoorthi G, Snyder C et al. Sequential anti-PD1 therapy following dendritic cell vaccination improves survival in a HER2 mammary carcinoma model and identifies a critical role for CD4 T cells in mediating the response. Front. Immunol. 10, 1939 (2019).

24. Fracol M, Datta J, Lowenfeld L et al. Loss of anti-HER-3 CD4+ T-helper T type 1 immunity occurs in breast tumorigenesis and is negatively associated with outcomes. Ann. Surg. Oncol. 24(2), 407-417 (2017).

25. Huang Y, Kim BYS, Chan CK, Hahn SM, Weissman IL, Jiang W. Improving immune-vascular crosstalk for cancer immunotherapy. Nat. Rev. Immunol. 18(3), 195-203 (2018).

26. Taylor CT, Colgan SP. Regulation of immunity and inflammation by hypoxia in immunological niches. Nat. Rev. Immunol. 17(12), 774-785 (2017).

27. Keating GM. Bevacizumab: a review of its use in advanced cancer. Drugs 74(16), 1891-1925 (2014).

28. Schmittnaegel M, Rigamonti N, Kadioglu E et al. Dual angiopoietin-2 and VEGFA inhibition elicits antitumor immunity that is enhanced by PD-1 checkpoint blockade. Sci. Transl. Med. 9(385), eaak9670 (2017).

29. Sierra JR, Corso S, Caione $\mathrm{L}$ et al. Tumor angiogenesis and progression are enhanced by Sema4D produced by tumor-associated macrophages. J. Exp. Med. 205(7), 1673-1685 (2008).

30. Smith ES, Jonason A, Reilly C et al. SEMA4D compromises blood-brain barrier, activates microglia, and inhibits remyelination in neurodegenerative disease. Neurobiol. Dis. 73, 254-268 (2015).

31. Evans EE, Jonason AS Jr, Bussler $\mathrm{H}$ et al. Antibody blockade of semaphorin $4 \mathrm{D}$ promotes immune infiltration into tumor and enhances response to other immunomodulatory therapies. Cancer Immunol. Res. 3(6), 689-701 (2015).

32. Evans EE, Paris M, Smith ES, Zauderer M. Immunomodulation of the tumor microenvironment by neutralization of semaphorin 4D. Oncoimmunology 4(12), e1054599 (2015).

33. Domblides C, Lartigue L, Faustin B. Control of the antitumor immune response by cancer metabolism. Cells 8(2), 104 (2019).

34. Kim S, Kim DH, Jung WH, Koo JS. Expression of glutamine metabolism-related proteins according to molecular subtype of breast cancer. Endocr. Relat. Cancer 20(3), 339-348 (2013).

35. Jiang N, Lin JJ, Wang J et al. Novel treatment strategies for patients with HER2-positive breast cancer who do not benefit from current targeted therapy drugs. Exp. Ther. Med. 16(3), 2183-2192 (2018).

36. Long JP, Li XN, Zhang F. Targeting metabolism in breast cancer: how far we can go? World J. Clin. Oncol. 7(1), 122-130 (2016).

37. Fischer K, Hoffmann P, Voelkl S et al. Inhibitory effect of tumor cell-derived lactic acid on human T cells. Blood 109(9), 3812-3819 (2007).

38. Wegiel B, Vuerich M, Daneshmandi S, Seth P. Metabolic switch in the tumor microenvironment determines immune responses to anti-cancer therapy. Front. Oncol. 8, 284 (2018).

39. Domblides C, Lartigue L, Faustin B. Metabolic stress in the immune function of t cells, macrophages and dendritic cells. Cells 7(7), 68 (2018).

40. Xu H, Zhang GX, Ciric B, Rostami A. IDO: a double-edged sword for T(H)1/T(H)2 regulation. Immunol. Lett. 121(1), 1-6 (2008).

41. Hwang SL, Chung NP, Chan JK, Lin CL. Indoleamine 2, 3-dioxygenase (IDO) is essential for dendritic cell activation and chemotactic responsiveness to chemokines. Cell Res. 15(3), 167-175 (2005).

42. Fallarino F, Grohmann U, Vacca C et al. T cell apoptosis by tryptophan catabolism. Cell Death Differ. 9(10), 1069-1077 (2002).

43. Marra A, Viale G, Curigliano G. Recent advances in triple negative breast cancer: the immunotherapy era. BMC Med. 17(1), 90 (2019). 
